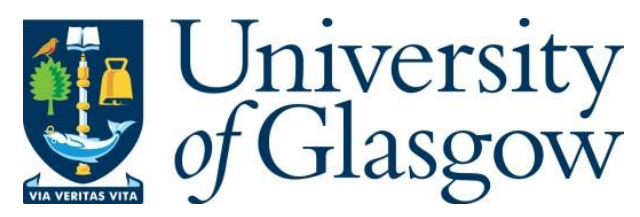

Deleau, C., Seat, H. C., Surre, F., Tap, H. and Bernal, O. D. (2021) Integrated widthmodulated SiN long period grating designed for refractometric applications. Journal of Lightwave Technology, 39(14), pp. 4820-4827.

There may be differences between this version and the published version. You are advised to consult the publisher's version if you wish to cite from it.

http://eprints.gla.ac.uk/247246/

Deposited on: 26 July 2021

Enlighten - Research publications by members of the University of Glasgow http://eprints.gla.ac.uk 


\title{
Integrated Width-Modulated SiN Long Period Grating Designed for Refractometric Applications
}

\author{
Clément Deleau, Member, IEEE, Han Cheng Seat, Senior Member, IEEE, Frederic Surre, Senior Member, IEEE, \\ Hélène Tap and Olivier D. Bernal, Member, IEEE
}

\begin{abstract}
A novel integrated photonic structure based on Long Period Waveguide Gratings (LPWGs) relying on channel-width modulation is proposed and tested for refractometric applications. These LPWGs have been fabricated through a Silicon Nitride process and experimentally characterized in terms of both surface and bulk sensitivities. Surface sensing configuration is first achieved by propagating one of the coupling optical modes into an epoxy-based negative photoresist SU8 cladding region that is in contact with the analyte via its outer surface. We subsequently show that the proposed LPWGs cladding layer can be advantageously replaced by a gas-porous polymeric bulk layer such as Styrene-co-AcryloNitrile (SAN) as the cladding region to be directly sensed to anticipate future gas sensing applications. Here, bulk sensing is optimized by increasing the analyte's influence on the modal propagation constants as it is demonstrated to be currently the most promising solution to effectively enhance the figure of merit of long period gratings of given lengths. Using varying water-glycerol mixtures, the surface sensitivity of these LPWGs has been measured at up to $240 \mathrm{~nm}$ per RI unit (RIU) that is in agreement with simulation. In addition, the bulk sensitivity has been indirectly estimated to be $\sim 1900 \mathrm{~nm} / \mathrm{RIU}$ via temperature measurements, which corroborates simulation results, thereby paving the way towards gas sensing applications.
\end{abstract}

Index Terms-Integrated photonics, long period grating, refractometry, coupled-local-mode theory, silicon photonics.

\section{INTRODUCTION}

$\mathbf{I}$ $\mathrm{N}$ recent years, integrated photonics has been intensely investigated for the development of highly sensitive labon-chip sensor applications. Due to their high degree of flexibility, they enable a multitude of functional designs to be implemented in a miniaturized circuit. The immunity of integrated photonic devices to electromagnetic and radiofrequency interferences coupled to their potential high resolution also render them very attractive [1] for in situ measurements and monitoring in harsh environments. Numerous embedded functions, once implemented, can thus be simultaneously driven or interrogated by a unique optical source on a thermally and mechanically robust platform. These characteristics have led to growing interest from the industry to develop and commercialize integrated optical sensors for indoor chemical monitoring, elevating the limits of detection towards everincreasing sensitivities. Integrated optical chemical sensors can detect and measure the concentration of an analyte by

C. Deleau, H. C. Seat, H. Tap and O. Bernal are with LAAS-CNRS, Université de Toulouse, CNRS, INP, 31000 Toulouse, France, e-mail: clement.deleau@laas.fr.

F. Surre is with James Watt School of Engineering, University of Glasgow, Glasgow, G12 8QQ, United Kingdom.

Manuscript received October XXX, 2020; revised XXX. accessing a particular optical parameter. Various designs for integrated refractometric measurements have been proposed over the years, including integrated interferometers [2], ring resonators [3], plasmonics [4], photonic crystals [5] and gratings [6]. These are principally reagent-mediated sensors [7], implying that they monitor the optical response of an intermediate species (typically a solid porous matrix) whose optical properties depend on the presence of the target gaseous analyte. This is advantageous in several ways; firstly, a very high selectivity to a particular analyte can be achieved since some material matrices or chemical transducers are uniquely responsive to a specific biochemical species, and secondly, it permits chemical interaction enhancement by focusing effort on optically optimizing and sizing of the material matrix. Amongst the three principal interrogation techniques, namely intensity-based measurements, wavelength or spectral interrogation and phase demodulation, wavelength-based techniques technically guarantee measurements which are relatively independent of any input intensity variations from the optical source.

In this work we propose a novel type of integrated refractometer using a planar multimode Long Period Grating (LPG). LPGs are structures operating on wavelength-selective coupling of two or more co-propagative modes whose effective indices can be analyzed by using the resonance spectral features. The LPG structure typically consists of a periodic longitudinal index or spatial modulation of a multi-mode supporting architecture with the modulation of the order of several times the wavelength to allow selective optical mode resonance. Historically, LPGs have been principally implemented on fiberbased platforms, as the introduction of such modulation can simply be performed by irradiation or tapering of the fiber core or cladding but have recently found interest in the integrated platform [8] [9]. In fiber LPGs, which couple core modes to cladding modes, the interaction coefficient between the optical fields and the external sensing region is usually very low, of the order of $0.001-0.01,(\sim 0.004)$ [10] for conventional fibers and slightly higher $(\sim 0.013)$ [11] for analyte-filled photonic crystal fibers. Nevertheless, the sensitivities that are obtained in these fiber-based devices are still within competitive levels. We hereby propose to exploit the design flexibility offered by integrated photonics in this work to create a highly sensitive and original chip-integrated sensor based on modal interaction optimization with the analyte of interest. Our design focuses on the coupling of the single core mode (in the silicon nitride layer) to cladding layer mode(s) by using periodic waveguide width-modulation as a pattern, as illustrated in Fig. 1. To 


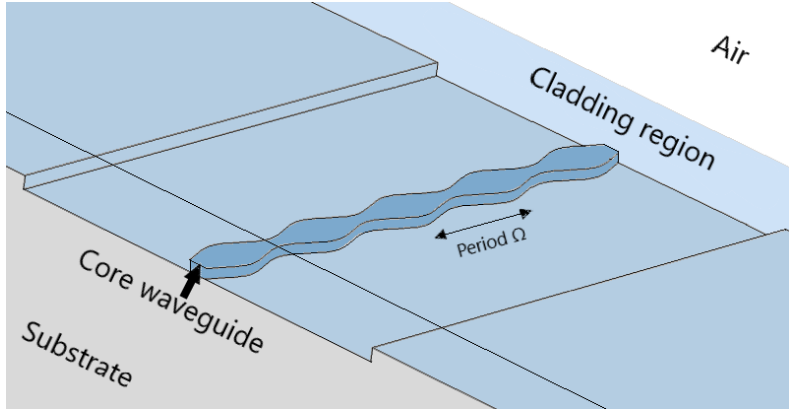

Fig. 1. Illustration of the proposed SiN integrated Long Period Grating

address future gas sensing applications, these LPGs could employ bulk sensing to benefit from the enhanced sensing matrix-optical field interaction with the cladding layer.

Section II describes the sensing scheme and structure features, and briefly discusses the different optimization methods that can be applied on an LPWG. The architecture's optical behavior is then modeled in Section III through EigenMode Expansion (EME) techniques whose advantages and limitations are discussed. Subsequently, Coupled-local-mode Theory (CLMT) [12] is introduced as a means to provide more insight on the optical behavior of the design and to facilitate coupling rate optimization. In Section IV, the fabrication and characterization of the LPWGs are presented, and the effects of the process variations on the optical system are analyzed in detail. In the last section, to assess the performance of the proposed LPWG, experimental and simulation results of the LPWG transmission spectrum and surface sensitivity are first presented using a cladding layer of epoxy-based negative photoresist SU8. Regarding bulk sensitivity, we will then show that it can be inferred from the temperature dependence tests. Finally, the SU8 cladding layer is replaced by a gas porous layer of Styrene-co-AcryloNitrile (SAN) that is demonstrated to be compatible with our LPG, thereby paving the way towards future gas sensing applications.

\section{DESIGN}

\section{A. Theory}

Long Period Gratings have been successfully exploited in Fiber Optic Chemical Sensors due to their ease of fabrication and, in particular, their capacity for coupling energy to outer cladding modes, thereby allowing the optical power to interact with the external environment. As duly reported in various papers [13] [14], a phase-matching condition between two co-propagative optical modes must be fulfilled to enable resonance to result in an exchange of energy :

$$
n_{e f f_{j}}-n_{e f f_{l}}=\frac{\lambda_{r e s}}{\Omega}
$$

where $n_{e f f_{j}}$ and $n_{e f f_{l}}$ are the effective indices (EIs) of the two coupled modes $j$ and $l$, respectively, $\lambda_{\text {res }}$ the center resonant wavelength and $\Omega$ the grating period. The formula is derived from the well-known Coupled Mode Theory (CMT) [12], from which coupling equations can be expressed as

$$
\frac{d b_{ \pm j}}{d z} \mp i \beta_{j} b_{ \pm j}=\sum_{j}\left(C_{ \pm j, l} b_{l}+C_{ \pm j,-l} b_{-l}\right)
$$

where $b_{ \pm j}$ is the modal amplitude of the forward $(+)$ or backward (-) propagating mode $j, \beta_{j}$ is the propagation constant of mode $j$, and $C_{j, l}$ is the mode coupling coefficient between modes $j$ and $l$. Then, straightforward development and conservation of energy show that when the mode coupling coefficients $C_{j, l}$ are periodically modulated along the longitudinal axis, with a modulation period corresponding to the accumulated phase difference between the two modes, coupling can occur. The rate of energy exchange upon resonance further depends on other parameters, including the modulation amplitude and optical field superposition at the modulated guide interfaces, as previously detailed in [15]. In refractometric sensing schemes employing LPGs, the presence of the analyte generally modifies the refractive index (RI) of the equivalent sensing layer which, in turn, modifies the propagation constants of the incident modes propagating in the layer. The difference in EI variation between the two coupled modes leads to a spectral shift of the optical resonance. This spectral shift can finally be measured to determine the RI variations of either the analyte deposited on top of the cladding layer (i.e. surface sensing configuration) or of the cladding layer itself (i.e. bulk sensing configuration). To a certain extent, in correlation with the modal overlap, which is defined as the modal optical field superposition ratio to the region of interest, the dependency of the EI of the $i^{t h}$ mode on the RI of the sensing layer $n_{\text {sens }}$ (used here as the cladding layer) can be expressed as the coefficient $\Gamma_{i}$ :

$$
\Gamma_{i}\left(\lambda, n_{e f f, i}\right)=\frac{d n_{e f f, i}}{d n_{\text {sens }}}\left(\lambda, n_{e f f, i}\right) .
$$

Now, by considering the phase-matching condition (equation (1)), the sensitivity of the LPG can thus be established as

$$
S=\frac{d \lambda_{\text {res }}}{d n_{\text {sens }}}=\lambda \frac{\Gamma_{j}\left(\lambda, n_{e f f, j}\right)-\Gamma_{l}\left(\lambda, n_{e f f, l}\right)}{n_{g, j}-n_{g, l}}
$$

where $n_{g, j}$ and $n_{g, l}$ are the group indices of the mode $j$ and $l$, respectively. Subsequently, the sensitivity of an LPG can be improved either by increasing the numerator term or decreasing the denominator term in equation (4). Based on the former, we propose to enhance the sensitivity of the LPWG by extensively increasing the $\Gamma_{l}$ coefficients by improving the modal overlap of the modes to couple in the sensing region. This could be achieved by inducing one of the modes to directly propagate in the sensing region, which is particularly applicable to gas sensors using gas-porous solid cladding layers. Moreover, due to the relatively large spectral width of the typical LPG resonance, to the order of several nanometers, the minimum detectable spectral shift is typically limited by the full-width half-maximum (FWHM) of the resonance instead of the optical analyser spectral resolution [16]. By using the CMT 


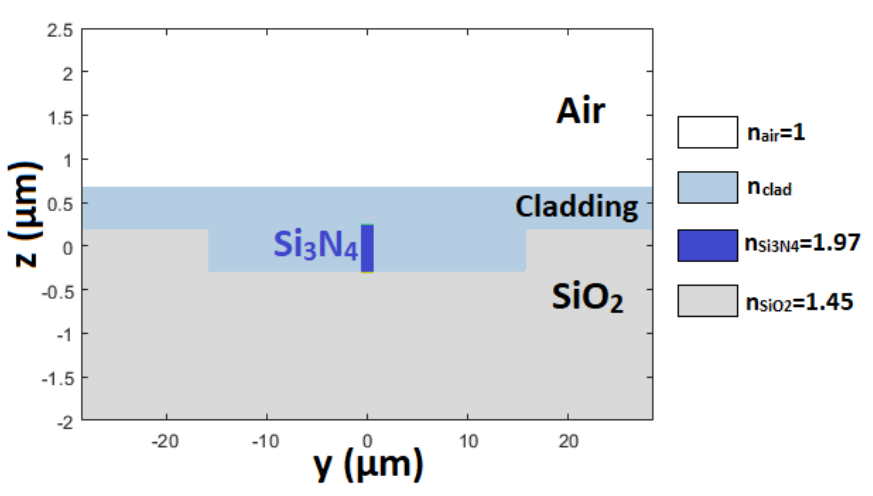

Fig. 2. Cross section material and refractive index profile of the proposed LPWG with $n_{\text {clad }}=1.58$ for SU8 and 1.57 for SAN

approach and taking into account modal dispersion effects, the figure of merit (FOM) can be expressed as follows :

$$
\text { F.O.M. }=\frac{S}{F W H M} \approx \frac{\left(\Gamma_{j}-\Gamma_{l}\right) L_{\text {opt }}}{0.8 \lambda_{\text {res }}}
$$

where $L_{\text {opt }}$ is the minimum LPG length for full mode coupling at resonance. The expression shows that for an LPG of a given length the FOM is ultimately limited by the coefficients $\Gamma_{j, l}$ and that tailoring of the spectral features can be performed by engineering $L_{\text {opt }}$ which depends on the coupling coefficients $C_{j, l}$, as will be detailed in the next section. Additionally, as it can be deduced from equations (4) and (5), improvements of the FWHM can be achieved by either reducing the coupling strength and proportionally increasing the grating length, or engineering the coupled modes propagation constants. The former method however decreases the optical transmission level with longer propagation in lossy integrated waveguides while the latter reduces the spectral sensitivity in proportion to the FWHM change, thus resulting in a constant FOM.

\section{B. Structure}

The proposed LPWG architecture consists of a Silicon Nitride $\left(\mathrm{Si}_{3} \mathrm{~N}_{4}\right)$ width-modulated core integrated waveguide and a multimode cladding waveguide, as shown in Fig 1. Smooth width modulation is advantageous as it allows continuous coupling, contrary to binary gratings where the waveguide is partly etched, thus potentially inducing loss of energy at the grating interfaces. In addition, the width modulation can be directly designed on the waveguide pattern, hence no additional process step is required.

The principal objective of our design is to obtain resonant coupling between the fundamental mode and a multi-mode in the cladding waveguide. As a main difference to fiber-based LPGs, whose cladding mode interaction with the sensing layer is generally very low, we elect to directly use the cladding cavity as the sensing layer, thus potentially increasing the coefficient $\Gamma_{l}$ to nearly 1 . To perform this operation, a polymeric layer of Styrene-co-AcryloNitrile (SAN) is selected for its important material properties pertaining to future potential gas sensing applications. Firstly, SAN has been previously proven as a suitable gas sensing host layer [2], and allows incorporation of selective reagents such as Cryptophane for functionalization to methane, for instance. Secondly, its RI $n_{S A N}(=1.57)$ is superior to the standard oxide material index of $n_{\mathrm{SiO}_{2}}=1.45$, thereby enabling the confinement of modes in a sufficiently large sensing layer without significant field leakage to the bottom oxide layer. It was also previously demonstrated as an acceptable integrated waveguide [17], and is thus expected to exhibit relatively minor diffractive imperfections which could arise due to the porosity of the polymer. Considering suitable cross-sectional dimensions, the following relationship between material RIs permits confinement of core and cladding modes: $n_{\text {core }}>n_{\text {eff }, 1}>n_{\text {clad }}>n_{\text {eff }, n}>$ $n_{\text {sub }}>n_{\text {air }}$ where the indices are respectively the RI of the core $n_{\text {core }}=n_{S i_{3} N_{4}}=1.97, n_{e f f, 1}$ the EI of the core's fundamental mode, $n_{\text {clad }}$ the RI of the cladding, $n_{\text {eff }, n}$ the EI of the $\mathrm{n}^{\text {th }}$ order cladding mode to couple, $n_{\text {sub }}$ the RI of the bottom $\mathrm{SiO}_{2}$ layer and $n_{\text {air }}$ the RI of air.

The SiN waveguide is designed at standard dimensions with a cross-section of $1000 \times 400 \mathrm{~nm}$ with a modulation width of $\pm 300 \mathrm{~nm}$ (see Fig. 2). Unlike waveguide Bragg grating reflectors whose periods are lower than the interrogating wavelength, waveguide LPGs could be subject to relatively higher energy loss at the period width minimas if the mode is too constrained. Preliminary experimental tests have, however, shown that no particular increase in optical loss is expected for both maximas or minimas of the waveguide width over a full modulation period. The cladding cavity dimensions are designed to be $1.1 \mu \mathrm{m}$ thick and $32 \mu \mathrm{m}$ wide, for a $1 \mathrm{~mm}$ long sensor, thus allowing to support multiple propagative cladding modes which can be coupled to the fundamental core waveguide mode.

\section{Modeling}

\section{A. Mode calculation and Eigenmode Expansion}

The waveguide structure supports a fundamental core mode, denoted by HE1, and multiple cladding modes (i.e. HE $i$ with $i=2,3,4 \ldots$, whose optical fields are shown in Fig. 3 for odd modes from HE7 to HE15. Each cladding mode possesses an EI slightly inferior to that of SAN that slowly decreases with the order of the mode. The cladding dimensions have been chosen to support up to eight hybrid odd HE modes which should result in eight resonant dips over a $200 \mathrm{~nm}$ spectral range. Moreover, as will be discussed in the next section, they also allow coupling parameters of different levels to be obtained which will translate into various dip amplitudes. As it is intended to couple the fundamental mode to cladding modes around $1550 \mathrm{~nm}$, the LPWG modulation period values are chosen to be between 8 and $10 \mu \mathrm{m}$, depending on the selected mode(s) to be coupled to. This is achieved by satisfying equation (1) and using the cross-section of the waveguide at zero-modulation amplitude for modal calculation. It is, however, important to understand that due to the nonlinear EI dispersion of the modes, the period-averaged EI is slightly different from the average width profile's EI, hence a small resonance shift can be expected between calculated (equation (1)) and simulated/experimental results. EigenMode Expansion (EME) is found to be an efficient solution for analyzing 


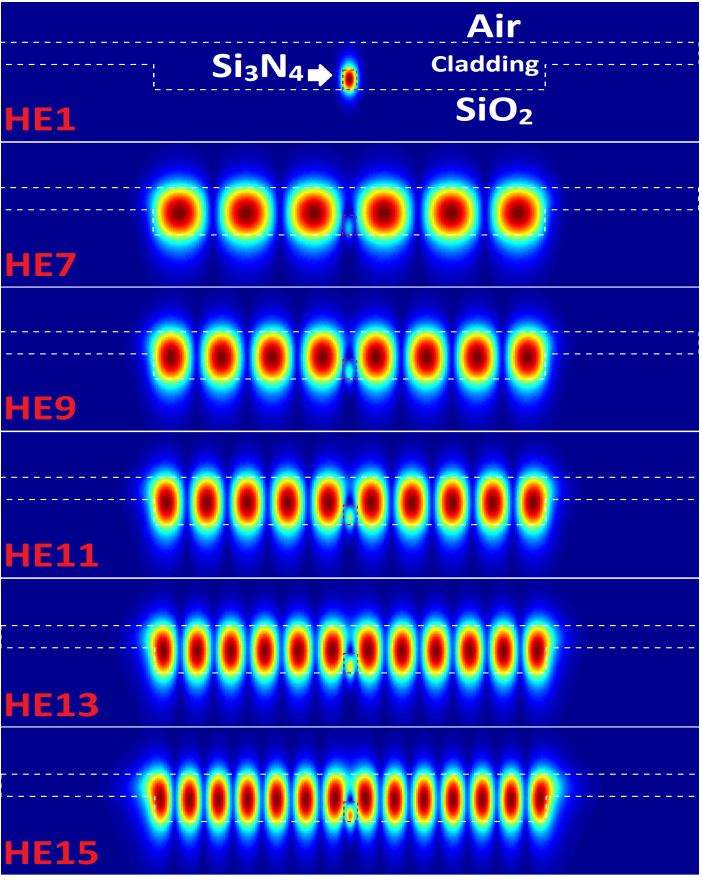

Fig. 3. Optical fields of cross-sectional odd modes

periodically-modulated structures. In EME, the structure is firstly divided into slices transverse to the axis of propagation. The optical fields and dispersion behaviors of the modes are then calculated for each cross-section after which a contingent iterative superposition of the scattering parameters is resolved to obtain the modulated modal information. Because of the post-mode-calculation analysis, which permits the setting of the structure's longitudinal profiles after the cross-sectional modal study, it is possible to optimize the LPWG in terms of length and periodicity with relative ease. Moreover, since EME studies the exchange of energy between modes, it is highly suitable for the design of our filter-type structure based on a multi-modal resonant coupling behavior. Figure 4 shows the output spectrum of the fundamental mode which exhibits multiple dips due to the different cladding mode resonances.

\section{B. Coupled-local-mode Theory approach}

Coupled-local-mode Theory (CLMT) provides an analytical model of the LPWG's response using parametric inputs such as its geometrical dimensions. Similar to EME, it requires the slicing of the structure and a modal calculation for each crosssection. However, mode coupling coefficients in CLMT are calculated analytically using the modulation parameters for each cross-section as shown in equation (6). The propagation calculation is then performed by the resolution of a set of differential equations as given in equation (2), contrary to CMT [12]. In addition, CLMT takes into account the important waveguide dimensional variations as well as polarization effects by using the coupling coefficient between the $j^{t h}$ and $l^{\text {th }}$ modes, denoted $C_{j, l}$, calculated for a large number of cross-

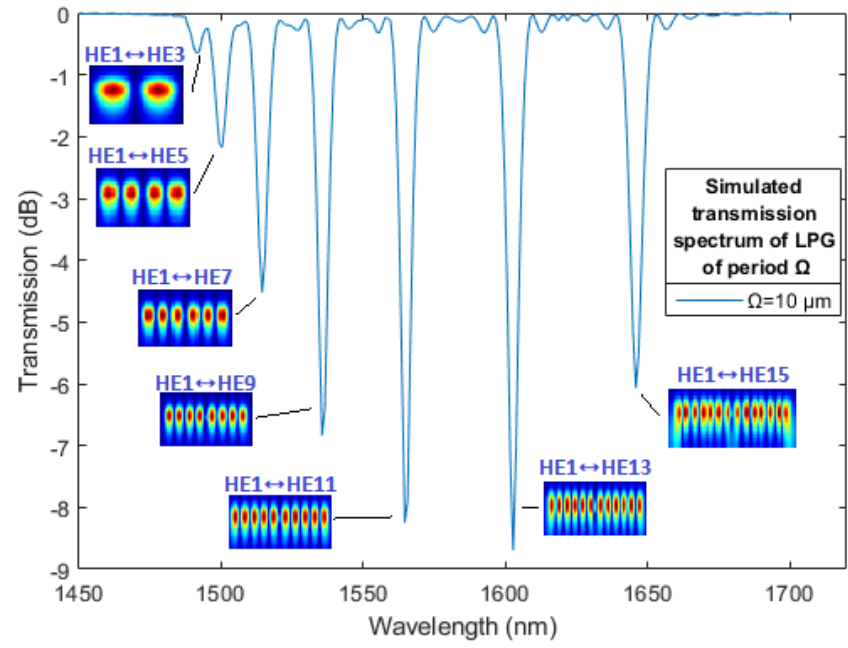

Fig. 4. Simulated transmission spectrum of 100-period LPWG showing coupling between fundamental and odd cladding modes. Insets illustrate various field profiles of coupled odd HEi modes

sections slices over a modulation period and defined by :

$$
\begin{aligned}
\forall j \neq l, \quad C_{j l} & =\frac{k}{4}\left(\frac{\varepsilon_{0}}{\mu_{0}}\right)^{\frac{1}{2}} \frac{1}{\beta_{j}-\beta_{l}} \int_{A_{\infty}} \hat{e}_{j}^{*} \hat{e}_{l} \frac{\partial n^{2}}{\partial z} d A, \\
C_{j j} & =0
\end{aligned}
$$

where $A_{\infty}$ is the infinite cross-section, $\epsilon_{0}$ and $\mu_{0}$ the freespace dielectric and permeability constants, respectively, $\Gamma$ the free-space wavenumber $2 \pi / \lambda$ and $*$ denotes the complex conjugate. The development of the coupling coefficients provides important information. First, through the differential RI integration over the modulation interfaces, it is seen that only odd modes can be coupled to the fundamental mode for a structure with vertical plane symmetry along the propagation axis: the calculated coefficient becomes null for even modes because of the opposite polarization states of the fields at the two modulated waveguide interfaces as described in detail in [15]. Secondly, the coupling length at resonance for total energy transfer can be adjusted by controlling the amplitude of the coupling coefficients. This can firstly be performed by optimizing the electric field superposition at the lateral corecladding interface. As an example, from Fig. 3, it is observed that the HE9 mode has a higher optical field strength at the core-cladding interface than the HE7 mode, hence a greater coupling coefficient is achieved. Additionally, the coupling strength also depends on the modulation amplitude. A deeper modulation thus leads to longitudinal shortening of the optimized sensor, but can also increase extrinsic waveguide loss with thinner waveguide widths or widen the resonance spectral pattern as described in equation (5). In Fig. 4, one also notices the tendency of the coupling dips of the higher-order mode (HE15) to be smaller, despite having a greater $\Gamma_{i}$ coefficient, as previously explained. This is because the energy starts coupling back to the fundamental mode after being exchanged completely; the exchange of energy is periodic. In most fiberbased LPGs, the coupling coefficients vary only slightly over a full period. As a result, the optimal grating length $L_{o p t}$ for the 


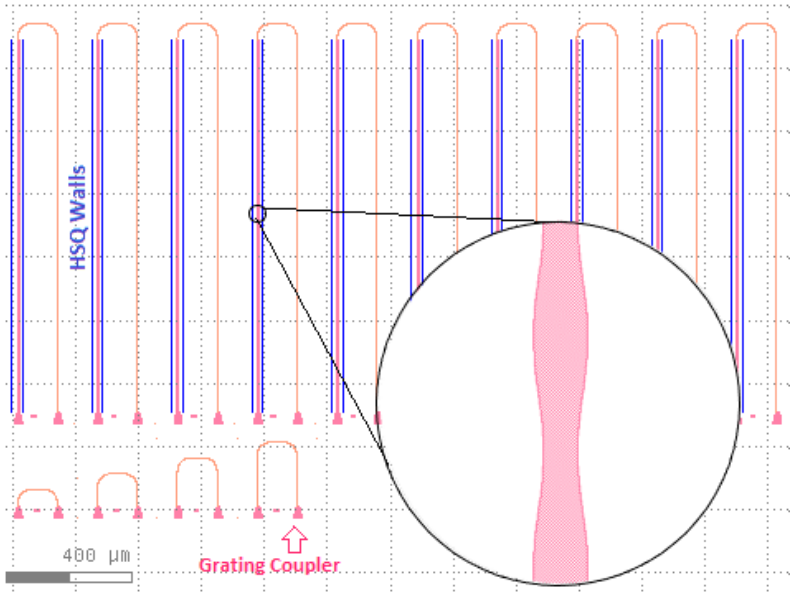

Fig. 5. LPWGs layout designed on Klayout

$j^{\text {th }}$ and $l^{\text {th }}$ mode coupling can be approximated by $\pi /\left(2 C_{j l}\right)$ [16]. However, for LPWGs, as previously demonstrated in [15], $L_{o p t}$ must be found numerically. This is due to larger variations of the coupling coefficient induced by typical profile modulations in integrated photonics.

\section{FABRICATION}

\section{A. Materials and Cross Section}

To validate the proposed LPWG architecture's operating principle and assess its surface sensitivity, a first set of LPWGs has been initially designed (Fig. 5) and fabricated using SU8 instead of SAN. Since both SU8 and SAN have very similar RIs and since SU8 has the particular advantage of being more easily deposited (in terms of flatness and thickness), SU8 is firstly used as cladding to investigate the operationality of the LPWG and characterize it as a prototype. Note that SU8 is an epoxy-based negative photoresist commonly used in photolithography, hence its optical and viscosity characteristics are widely known. This enables a greater control to be achieved on the cladding layer. It is, however, not porous to gaseous species and is only used here to validate the operating optical principle. For simplicity of fabrication, the cladding is delimited by incorporating $\mathrm{SiO}_{2}$ walls using Hydrogen Silesquioxane (HSQ) resist, whose constitution and $\mathrm{RI}$ are found to be very similar to those of $\mathrm{SiO}_{2}$ after curing. The HSQ walls are designed to be $3 \mu \mathrm{m}$ wide and $0.6 \mu \mathrm{m}$ thick to prevent multimode optical leakage as well as to ensure ease of coating and electron-patterning.

\section{B. Process}

To fabricate our waveguide, a $2 \mu \mathrm{m} \mathrm{SiO}_{2}$ layer is first deposited by plasma-enhanced chemical vapor deposition (PECVD) on a clean Si wafer. Parasitic substrate optical leakage during waveguide modal propagation has been previously investigated for this material thickness and found to be negligible. A $400 \mathrm{~nm}$ thick $\mathrm{Si}_{3} \mathrm{~N}_{4}$ (or simply $\mathrm{SiN}$ ) layer is next deposited on top by PECVD, with $n_{\text {core }}(\sim 1.97)$ measured by ellipsometry. An electron-sensitive resist MaN2405 is deposited by spin-coating, followed by an Electra92 resist layer,

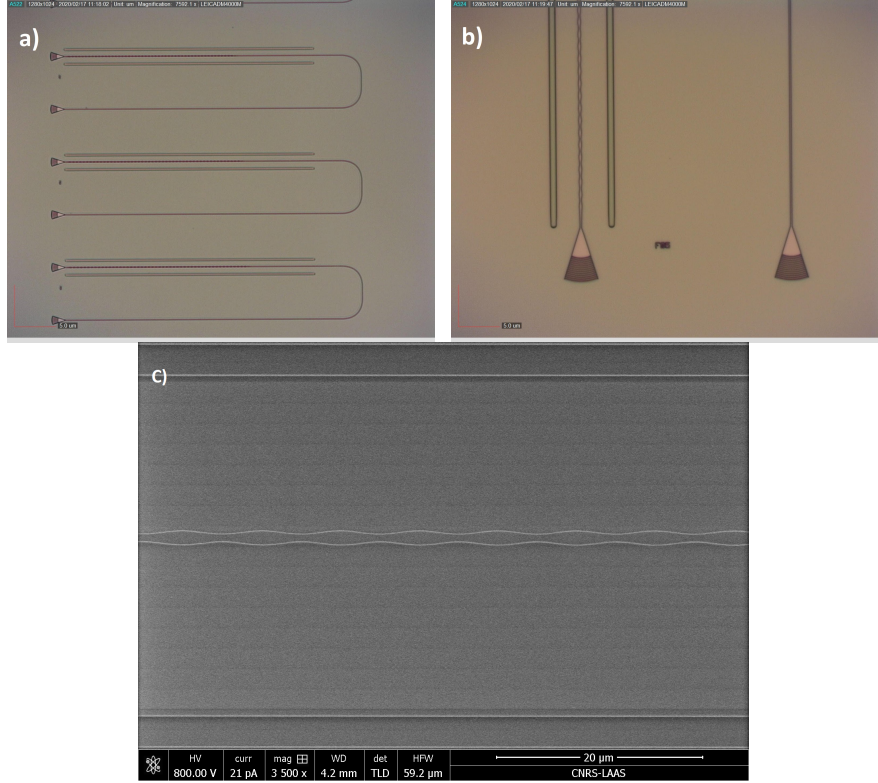

Fig. 6. Fabricated LPWGs die microscope photographies: (a) overview, (b) grating couplers zoom and (c) SEM image of the grating modulation

principally used for the dissipation of electronic charges that are induced during the electron-beam lithography stage. After development of the waveguide patterns, the unwanted parts of the SiN layer are removed by reactive ion etching (RIE). The remaining parts of the resist are then removed using acetone and Plasma $\mathrm{O}_{2}$ cleaning. Both the HSQ and Electra92 resists are then deposited by spin-coating. After curing of the HSQ walls, a final layer of SU8 is deposited by spin-coating at a spin-rate of $1700 \mathrm{rpm}$. This layer amounts to $1.1 \mu \mathrm{m}$ thick as measured by profilometry. Figure 6 shows optical microscope pictures and a zoomed scanning electron microscope (SEM) image of the fabricated LPWGs.

\section{Post-Fabrication Characterization}

Scanning electron microscopy has revealed a slight asymmetry of the structure, due to a minor translation shift between the two patterned layers. As will be shown in the next section, this asymmetry has only minor consequences on the output spectrum as well as on the sensor's sensitivity. The measured waveguide width has also been found to be slightly different from the design, which should induce a small resonance shift in the transmission spectrum since it slightly modifies the average EI of the core mode.

\section{EXPERIMENTAL RESULTS}

\section{A. Experimental Setup}

The experimental setup for characterizing our structure consists of a broadband light source (BBS), an optical spectrum analyzer (OSA), a fiber array and the chip under test, as illustrated in Fig. 7. The fiber array incorporates a set of multiple fibers bound and held together by a support at one end, with each fiber core separated by $127 \mu \mathrm{m}$. Each structure to be characterized is connected at each respective output to a grating coupler, which allows the injection and recovery of 


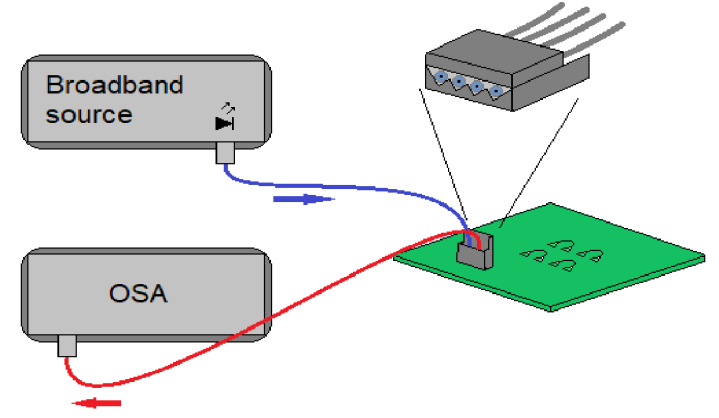

Fig. 7. Illustration of the experimental setup

the interrogating light through the fiber array positioned on top of the chip-integrated grating coupler pair. The fiber array is mounted onto a motorized linear stage driven by a dedicated LabVIEW program for alignment purposes. An algorithm has also been incorporated into the program to enable automatic scanning of the entire chip via feedback of the measured transmission spectra of the components or functions under characterization. For temperature stability, the chip is placed on top of a Thorlabs mount with integrated thermo-electric cooler.

\section{B. Virtual Reconstruction Of The Entire Coupling Spectrum}

As shown in equation (1), the position of the resonance peak due to coupling resonance within the structure depends proportionally on the period of the LPWG for a given crosssection. Furthermore, Fig. 4 shows that the theoretical transmission spectrum of the structure containing the first-order coupling resonance dip of every cladding mode is $\sim 200 \mathrm{~nm}$ wide and too large to be measured over a single wavelength scan by our setup. Hence, a set of LPWGs with different periods but identical cross-section and modulation amplitude has been designed and fabricated instead. It then becomes possible to virtually reconstruct the entire spectrum by firstly de-embedding the signal to isolate the LPWG's individual spectral contribution and, secondly, by shifting the processed signals respectively to the corresponding LPWGs periods. The LPWGs exhibit little or no additional energy loss when compared to non-modulated waveguides of equal length. After base-line correction, the LPWGs transmissions are shown on the reconstructed spectrum in Fig. 8(b). Subsequently, when fabrication defaults such as asymmetry or waveguide width defects are reintroduced into the model, a very similar spectra can be found as in 8(a).

Contrary to Fig. 4, shorter dips (1-3dB) have been observed between the main dips $(3-10 \mathrm{~dB})$. They may be attributed to the slight asymmetry of the structure which disrupts the nullification condition of the optical modal field product at the lateral interfaces for even modes. This consequently leads to a minor rise in the coupling coefficients of the even modes, thus allowing partial coupling to these same even modes. Nonetheless, a flaw in the virtual spectrum reconstruction is highlighted by the differing measured amplitudes for the same coupling dips and can be attributed to the high spectral
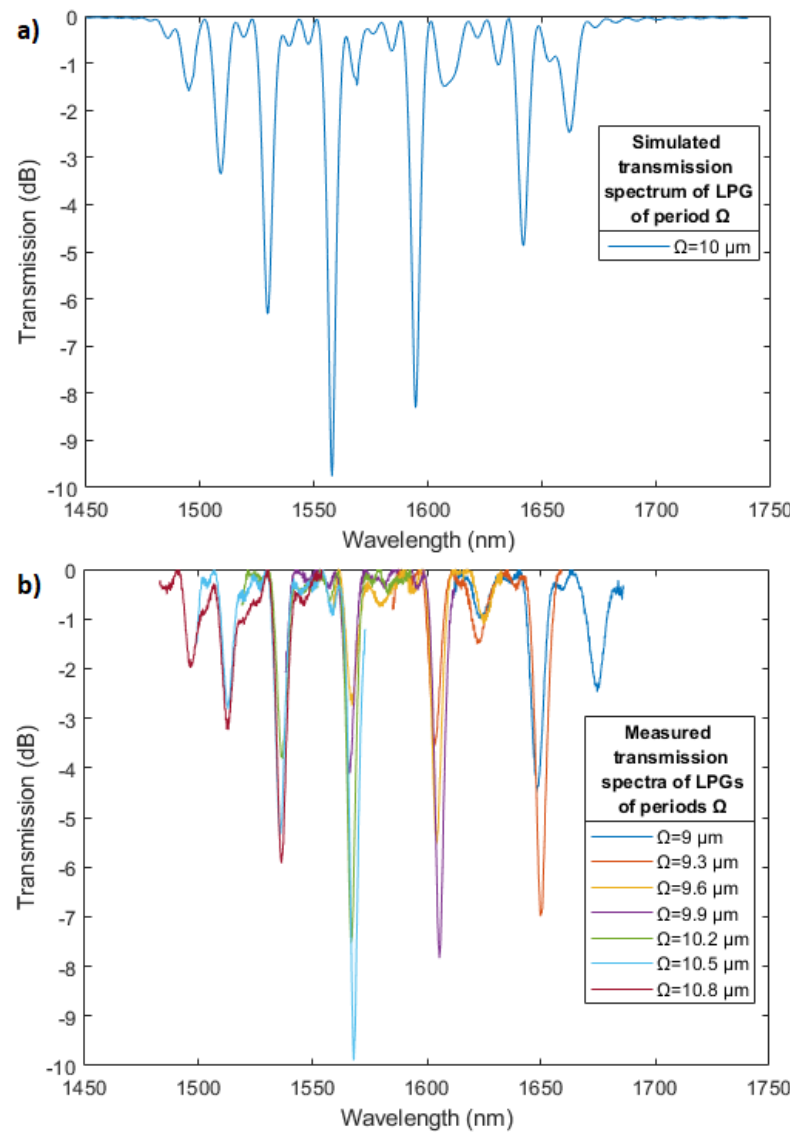

Fig. 8. Transmission spectra for a 100-period long LPWG: (a) Simulated with parametric adjustment and (b) reconstructed from measurements

dispersion of the modal coupling coefficients in high indexcontrast gratings. This is because with higher wavelengths, the fundamental mode becomes more leaky and results in large variations of the optical field product at the waveguide's modulated interfaces, thereby changing the coupling strength. The different coupling dips are found to exhibit extinction ratios of several dBs. The dips also exhibit an average FWHM of approximately $5 \mathrm{~nm}$ in this work. Using the SANTEC tunable laser (TSL-550 C model) with its swept test system, which has a $\pm 1 \mathrm{pm}$ repeatability and resolution, 200 wavelength scan measurements have been performed over an hour with a regulated temperature of $23^{\circ} \mathrm{C}$ to estimate the precision $\sigma_{\text {sensor }}$ of the resonance wavelength of the HE9 mode's transmission spectrum. After applying a Lorentzian curve fitting, $\sigma_{\text {sensor }}=1.5 \mathrm{pm}$ is obtained, which is similar to the laser system precision.

\section{Refractometric measurements}

A cladding refractometric measurement configuration is difficult to achieve here since a gas-porous sensing region is a solid layer which cannot be filled or saturated with liquids. We thereby propose a simple surface-based experimental configuration where the target liquid is poured onto the top surface of the sensor. Because of the relatively thin SU8 cladding, $\Gamma_{l}$ is of the order of 0.1 which remains higher than the coefficients of the cladding modes in most fiber LPGs, 


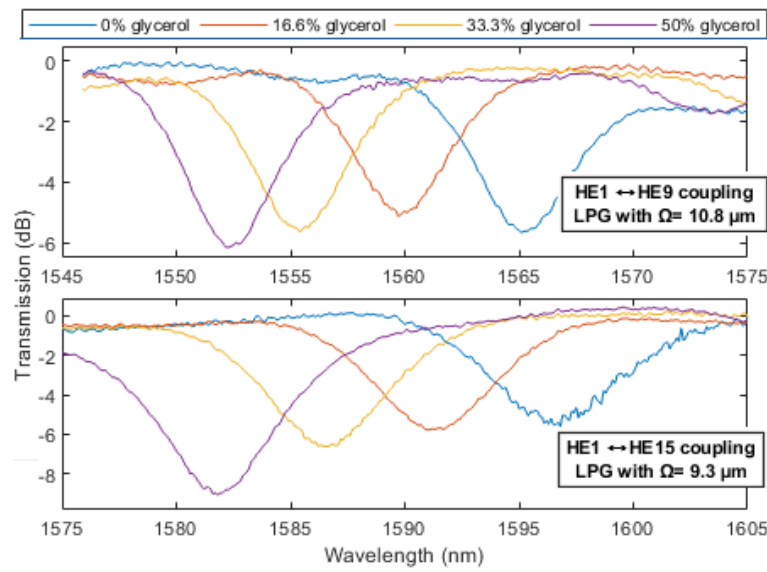

Fig. 9. Measured spectrum of HE9 and HE15 mode resonance of LPWGs using SU8 as cladding for different deposited concentrations of water and glycerol

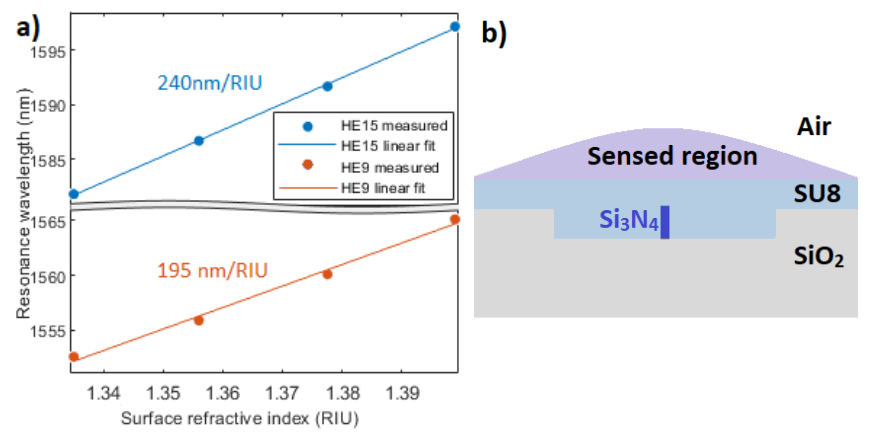

Fig. 10. (a) Measured surface spectral sensitivities of modes HE9 and HE15 and (b) Surface sensing configuration illustration

when the surface of the structure becomes the sensing region. Water and glycerol mixtures of different proportions are then applied on different LPWGs and the resonance shifts of mode coupling dips tracked, as shown in Fig. 9 for the HE9 and HE15 modes. Using the RIs of water and glycerol around $1550 \mathrm{~nm}$ [18], the surface sensitivities are measured (Fig. 10) and compared to simulation, for both surface and cladding measurements in Table I. A sensitivity up to $240 \mathrm{~nm} / \mathrm{RIU}$ has been measured for the HE15 mode's resonance dip, whose bandwidth and extinction ratio values are both found to agree with the simulated spectrum. Additionally, it is observed that when fleeting impurities interact with the cladding layer during measurement, the resonance features are not shifted, due to the inertial behavior of the LPWG. Because the modal energy exchange during propagation is driven by a coupling momentum, the LPWG sensor's resonance wavelength depends on the sensed layer's median RI and not the average RI, thus rendering it robust to disturbance by impurities. Additionally, a cross-sectional modification on a small segment of the LPWG does not lead to any significant shift in the LPWG resonance but can, however, contribute to reduce the coupled energy, making LPWGs highly interesting candidates for sensing of gases whose RI profiles are generally homogeneous within a given volume. As the measured and simulated sensitivities are found to be very similar for the surface measurement configuration, the experimental measurements are taken to corroborate the model and the subsequent simulations without loss of generality, thus validating the operation of our sensor with data-processed inputs. The comparison also suggests that the cladding measurements can further be expected to conform to the simulated values. Using our measured sensor resolution, a detection limit of $2 \mu \mathrm{RIU}$ in the case of bulk sensing can be estimated.

TABLE I

CALCUlATED AND MEASURED SENSITIVITIES

\begin{tabular}{|c||c|c|c|}
\hline & $\begin{array}{c}\text { Simulated } \\
\text { surface } \\
\text { sensitivity } \\
\text { (nm/RIU) }\end{array}$ & $\begin{array}{c}\text { Measured } \\
\text { surface } \\
\text { sensitivity } \\
\text { (nm/RIU) }\end{array}$ & $\begin{array}{c}\text { Simulated } \\
\text { bulk } \\
\text { sensitivity } \\
\text { (nm/RIU) }\end{array}$ \\
\hline \hline HE9 & 198 & 195 & 1920 \\
\hline HE11 & 214 & 206 & 1915 \\
\hline HE13 & 231 & 227 & 1910 \\
\hline HE15 & 251 & 240 & 1820 \\
\hline
\end{tabular}

\section{Temperature dependence}

The temperature behavior of the sensor is firstly simulated with EME using thermo-optical coefficients of $\mathrm{SiN}, \mathrm{SiO}_{2}$ and SU8, whose values at $1550 \mathrm{~nm}$ are respectively $\sim 24.5,9.5$ [19] and $-130 \mu \mathrm{RIU} / \mathrm{K}$ [20]. The temperature sensitivity of the wavelength resonances is estimated to be $\sim 330 \mathrm{pm} / \mathrm{K}$ and is similar for each mode resonance. For experimental validation, the TEC temperature is swept from 15 to $30^{\circ} \mathrm{C}$ at $5^{\circ} \mathrm{C}$ steps. Linear interpolation of the measured values gives a temperature sensitivity of $340 \mathrm{pm} / \mathrm{K}$ as plotted in Fig. 11 . In addition, from the temperature dependence analysis, an approximation of the bulk sensitivity can be inferred. This is estimated to be $\sim 1900 \mathrm{~nm} / \mathrm{RIU}$.

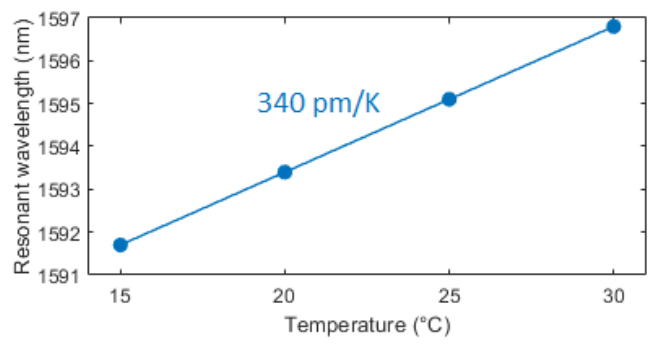

Fig. 11. Measured temperature dependence of the wavelength resonance

\section{E. SAN coating}

As previously mentioned, SU8 cannot, in practice, be used as a gas sensing layer as it is a hermetic layer. Instead, a porous polymeric layer of similar RI such as SAN can be used for gas sensing applications [2]. To ensure SAN compatibility with the proposed design architecture as the cladding, coating and characterization are performed on SAN as follows: $50 \mathrm{mg}$ of SAN are dissolved in $10 \mathrm{~mL}$ of methylethylketone and the mixture is spin-coated onto the pre-cleaned photonic chip. As seen in Fig. 12, the coupling phenomenon occurs very similarly to that of the SU8-coated LPWG. Small changes of 


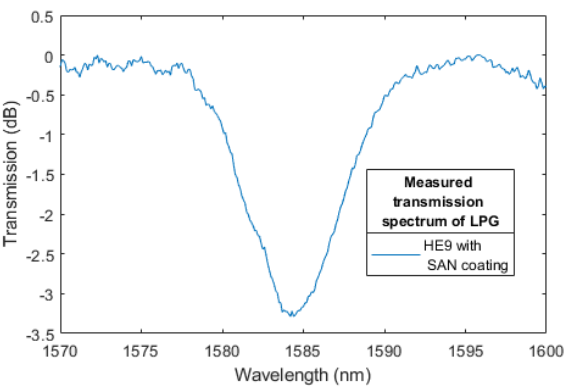

Fig. 12. Measured transmission spectrum of HE9 mode resonance on LPWG with SAN cladding

coupling are due to minor variations in the film thickness and layer RI deviation as compared to the previously-characterized LPWGs. Consequently, employing SAN as an external sensing layer provides a pathway to gas sensing based on the bulk sensitivity of the proposed LPWG. In addition, since the thermo-optical coefficients of SAN and SU8 are of the same order [21], the thermal behavior in a SAN-clad LPWG is not expected to significantly vary from the presented results.

\section{CONCLUSION}

In this work, SiN width-modulated LPWGs have been designed, fabricated and characterized to achieve RI measurements with high sensitivity. Surface sensitivities up to $240 \mathrm{~nm} / \mathrm{RIU}$ have been attained and agree with both the proposed CLMT model and EME simulations. In addition, from temperature tests, the bulk sensitivity has been estimated to be approximately $1900 \mathrm{~nm} / \mathrm{RIU}$, in accordance with EME simulations. This is an improvement of almost an order of magnitude and demonstrates possibly a very high sensitivity for potential gas sensing applications. A specific use of SAN's high RI is demonstrated to allow modal propagation, which could improve both the sensitivity and FOM of our sensor by eight-fold based on a bulk measurement configuration. The results from this work will serve as a baseline for the design of LPWGs combining modal interaction optimization with other sensitivity enhancement techniques such as phase-matching turning point optimization [14].

\section{ACKNOWLEDGMENT}

The fabrication and characterization of the integrated photonic long period gratings are supported by a TTIL (Toulouse Tech Interlabs) 2019 grant and by LAAS-CNRs micro and nanotechnologies platform, a member of the Renatech french national network. The authors would like to thank A. Lecestre, F. Carcenac, J-B. Doucet, L. Mazenq and P-F. Calmon for their technical support during the fabrication and characterization processes of the photonic integrated circuits.

\section{REFERENCES}

[1] L. Chrostowski and M. Hochberg, Silicon Photonics Design: From Devices to Systems. Cambridge University Press, 2015.

[2] F. T. Dullo, S. Lindecrantz, J. Jágerská, J. H. Hansen, M. Engqvist, S. A Solbø, and O. G. Helles $\varnothing$, "Sensitive on-chip methane detection with a cryptophane-a cladded mach-zehnder interferometer," Opt. Express, vol. 23, no. 24, pp. 31564-31 573, Nov 2015. [Online]. Available: http://www.opticsexpress.org/abstract.cfm?URI=oe-23-24-31564
[3] N. A. Yebo, S. P. Sree, E. Levrau, C. Detavernier, Z. Hens, J. A Martens, and R. Baets, "Selective and reversible ammonia gas detection with nanoporous film functionalized silicon photonic micro-ring resonator," Opt. Express, vol. 20, no. 11, pp. 11 855-11 862, May 2012. [Online]. Available: http://www.opticsexpress.org/abstract.cfm?URI=oe20-11-11855

[4] A. Zaki, K. Kirah, and M. Swillam, "Integrated optical sensor using hybrid plasmonics for lab on chip applications," Journal of Optics, vol. 18 , p. $085803,082016$.

[5] M. Danaie and B. Kiani, "Design of a label-free photonic crystal refractive index sensor for biomedical applications," Photonics and Nanostructures - Fundamentals and Applications, vol. 31, pp. 89 - 98, 2018. [Online]. Available: http://www.sciencedirect.com/science/article/pii/S1569441017303735

[6] S. Jain, S. Srivastava, S. Rajput, L. Singh, P. Tiwari, A. K. Srivastava, and M. Kumar, "Thermally stable optical filtering using silicon-based comb-like asymmetric grating for sensing applications," IEEE Sensors Journal, vol. 20, no. 7, pp. 3529-3535, 2020.

[7] C. McDonagh, C. S. Burke, and B. D. MacCraith, "Optical chemical sensors," Chemical Reviews, vol. 108, no. 2, pp. 400-422, 2008, pMID: 18229950. [Online]. Available: https://doi.org/10.1021/cr068102g

[8] Q. Liu, K. S. Chiang, and K. P. Lor, "Condition for the realization of a temperature-insensitive long-period waveguide grating," Opt. Lett., vol. 31, no. 18 , pp. 2716-2718, Sep 2006. [Online]. Available: http://ol.osa.org/abstract.cfm?URI=ol-31-18-2716

[9] U. Tripathi and V. Rastogi, "Temperature insensitive long period waveguide gratings in rib waveguide," Optik, vol. 186, pp. $15-21,2019$. [Online]. Available: http://www.sciencedirect.com/science/article/pii/S0030402619305406

[10] E. Simões, I. Abe, J. Oliveira, O. Frazão, P. Caldas, and J. Pinto, "Characterization of optical fiber long period grating refractometer with nanocoating," Sensors and Actuators B: Chemical, vol. 153, no. 2, pp. 335 - 339, 2011. [Online]. Available: http://www.sciencedirect.com/science/article/pii/S0925400510008518

[11] H. Hu, C. Du, Q. Wang, X. Wang, and Y. Zhao, "High sensitivity internal refractive index sensor based on a photonic crystal fiber long period grating," Instrumentation Science \& Technology, vol. 45, no. 2, pp. 181-189, 2017. [Online]. Available: https://doi.org/10.1080/10739149.2016.1215994

[12] A. W. Snyder and J. Love, Optical waveguide theory. Springer Science \& Business Media, 2012.

[13] L. Jin, W. Jin, J. Ju, and Y. Wang, "Coupled local-mode theory for strongly modulated long period gratings," Journal of Lightwave Technology, vol. 28, no. 12, pp. 1745-1751, 2010.

[14] R. Y.-N. Wong, D. H. J. Juan, M. Ibsen, and P. P. Shum, "Optical fibre long-period grating sensors operating at and around the phase matching turning point," Applications of Optical Fibers for Sensing, 012019.

[15] C. Deleau, H. Seat, H. Tap, F. Surre, and O. Bernal, "Integrated silicon nitride horizontal long period grating for refractometric gas sensing applications," in 2020 IEEE International Instrumentation and Measurement Technology Conference (I2MTC), 2020, pp. 1-6.

[16] L. Rindorf and O. Bang, "Sensitivity of photonic crystal fiber grating sensors: Biosensing, refractive index, strain, and temperature sensing," Journal of the Optical Society of America B, vol. 25, 082007.

[17] G. Qureshi, N. Padha, V. Gupta, M. Kamalasanan, A. Singh, A. Kapoor, and K. Tripathi, "Styrene acrylonitrile (san) based polymeric waveguides," Optics and Laser Technology, vol. 35, no. 5, pp. 401 - 407, 2003. [Online]. Available: http://www.sciencedirect.com/science/article/pii/S0030399203000379

[18] J. E. Saunders, C. Sanders, H. Chen, and H.-P. Loock, "Refractive indices of common solvents and solutions at 1550\&\#x2009;\&\#x2009;nm,” Appl. Opt., vol. 55, no. 4, pp. 947-953, Feb 2016. [Online]. Available: http://ao.osa.org/abstract.cfm?URI=ao55-4-947

[19] A. Arbabi and L. Goddard, "Measurements of the refractive indices and thermo-optic coefficients of si3n 4 and siox using microring resonances," Optics Letters, vol. 38, 102013.

[20] M. Ibrahim, J. Schmid, A. Aleali, P. Cheben, J. Lapointe, S. Janz, P. Bock, A. Densmore, B. Lamontagne, R. Ma, D. Xu, and W. Ye, "Athermal silicon waveguides with bridged subwavelength gratings for te and tm polarizations," Optics express, vol. 20, pp. 18 356-61, 072012.

[21] N. G. Sultanova, S. N. Kasarova, and I. D. Nikolov, "Characterization of optical properties of optical polymers," Optical and Quantum Electronics, vol. 45, no. 3, pp. 221-232, Mar 2013. [Online]. Available: https://doi.org/10.1007/s11082-012-9616-6 